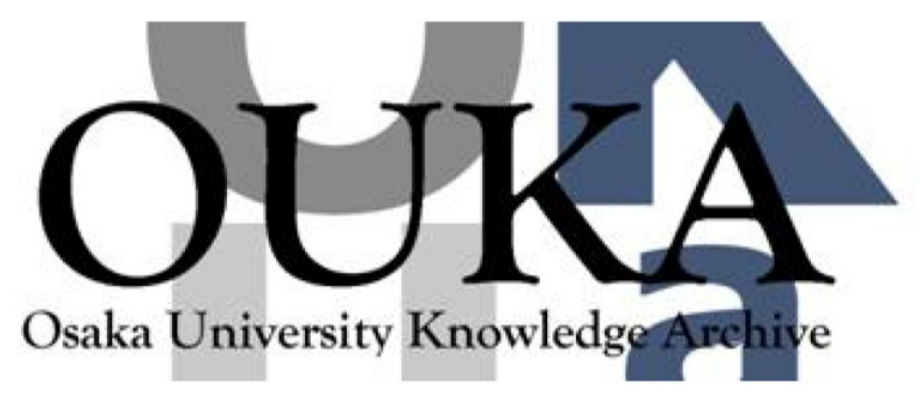

\begin{tabular}{|c|c|}
\hline Title & $\begin{array}{l}\text { Broadband radio interferometer utilizing a } \\
\text { sequential triggering technique for locating } \\
\text { fast-moving electromagnetic sources emitted } \\
\text { from lightning }\end{array}$ \\
\hline Author(s) & Mardiana, Redy; Kawasaki, Zen \\
\hline Citation & $\begin{array}{l}\text { IEEE Transactions on Instrumentation and } \\
\text { Measurement. } 49(2) \text { p. } 376-p .381\end{array}$ \\
\hline Issue Date & $2000-04$ \\
\hline oaire:version & VoR \\
\hline URL & https://hdl. handle. net/11094/3418 \\
\hline rights & $\begin{array}{l}\text { ब2000 IEEE. Personal use of this material is } \\
\text { permitted. However, permission to } \\
\text { reprint/republish this material for advertising } \\
\text { or promotional purposes or for creating new } \\
\text { collective works for resale or redistribution } \\
\text { to servers or lists, or to reuse any } \\
\text { copyrighted component of this work in other } \\
\text { works must be obtained from the IEEE.. }\end{array}$ \\
\hline Note & \\
\hline
\end{tabular}

Osaka University Knowledge Archive : OUKA

https://ir. Library. osaka-u. ac. jp/

Osaka University 


\title{
Broadband Radio Interferometer Utilizing a Sequential Triggering Technique for Locating Fast-Moving Electromagnetic Sources Emitted from Lightning
}

\author{
Redy Mardiana, Member, IEEE, and Zen Kawasaki, Member, IEEE
}

\begin{abstract}
A broadband radio interferometer to investigate the location of fast-moving electromagnetic (EM) sources emitted from lightning discharges has been designed. A sequential triggering technique was applied to the system for recording the data of broadband electromagnetic pulses from 25 to $250 \mathrm{MHz}$. Once the electromagnetic pulse is detected and its amplitude exceeds a threshold level, the triggering circuit is turned on to record the waveform and get ready to acquire another pulse afterwards. This technique can overcome the limitation of the digitizer's memory due to such high digitization rate and very large bandwidth. We have implemented our system to locate and retrace fast-moving electromagnetic sources emitted from lightning discharges during a field experiment in Australia in summer 1997. As one of the results, a cloud-to-ground lightning discharge has been reconstructed in the two-spatial dimension and in time sequence.
\end{abstract}

Index Terms-Broadband interferometer, electromagnetic radiation, interferometer, lightning, sequential trigger.

\section{INTRODUCTION}

$\mathbf{M}$ ORE THAN two decades ago, the time-of-arrival (TOA) and narrow-band interferometer systems were first developed to study the dynamics of lightning. Both systems show excellent results for mapping lightning radiation and can explain lightning progression in detail [1]-[7]. Currently we have developed another system based on an interferometer but using a broadband signal processing. Unlike the narrow-band interferometer, which is operated at a fixed frequency to estimate the source positions, the broadband interferometer takes into account all frequency components of the lightning flash emitted during its progression and uses them to resolve the source positions.

It is known that electromagnetic (EM) radiation from lightning flashes is characterized by a broadband spectrum from a few $\mathrm{kHz}$ through several $\mathrm{GHz}$, and during the occurrence of a lightning flash broadband EM radiation is emitted continuously [8]. Since the lightning spectrum has very large bandwidth, the broadband interferometer system needs a high digitization rate to record its broadband signals, but since the memory of the digitizer is limited, the entire radiation from a lightning flash cannot

Manuscript received May 26, 1999; revised February 2, 2000.

The authors are with the Department of Electrical Engineering, Faculty of Engineering, Osaka University, Suita, Osaka 565, Japan.

Publisher Item Identifier S 0018-9456(00)03429-X.

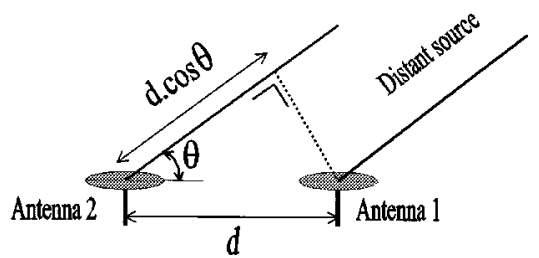

Fig. 1. Two broadband antenna sensors separated horizontally.

be recorded continuously. Also, it is not practical if the digitizer has to record undetected broadband pulses during a lightning flash progression. To overcome the above disadvantage, a sequential triggering technique was proposed where the instrumentation begins to acquire data only if a lightning flash emits broadband pulses exceeding a threshold level. Shao et al. [9] have illustrated the basic principle of a broadband interferometer and used this technique in the one-spatial dimension. Ushio et al. [10] and Mardiana et al. [11] reported more clearly the use of a broadband interferometer for locating fast-moving electromagnetic sources with $500 \mathrm{MHz}$ sampling rate of the digitizer and mapping the two-spatial and temporal progression of lightning discharge processes.

In this paper we give particular attention to the detail principle of our broadband interferometer system and the experimental setup along with the sequential triggering technique. A short report of one of our experimental results will also be discussed.

\section{DETAIL OF A BROADBAND INTERFEROMETER}

\section{A. Basic Principle}

The basic idea of this interferometer technique is to estimate the phase differences at various frequency components of Fourier spectra between a pair of broadband antenna sensors. The simplest radio interferometer consists of two separate receiving antenna sensors. Consider two broadband antenna sensors separated horizontally above the ground by a distance $d$, as shown in Fig. 1. The broadband signal which is originated from a common source impinging on antenna 1 is $r_{k}(t)$ and on antenna 2 is $r_{l}(t)$. Assuming a total record length of $T$, then deriving the signals in the frequency domain by using Fourier transformation [9]-[12], we have

$$
R_{k}(f)=\int_{0}^{T} r_{k}(t) e^{-j 2 \pi f t} d t
$$




$$
R_{l}(f)=\int_{0}^{T} r_{l}(t) e^{-j 2 \pi f t} d t
$$

The cross correlation between both signals is related to the cross energy spectral density function by the well-known relationship

$$
G_{k l}(f)=R_{k}^{*}(f) \cdot R_{l}(f)
$$

where $*$ denotes the complex conjugate [12]. The $G_{k l}(f)$ will generally be a complex number such that

$$
G_{k l}(f)=G_{\operatorname{Re}}(f)-j G_{\operatorname{Im}}(f)=\left|G_{k l}(f)\right| e^{-j \phi(f)}
$$

where

$$
\begin{aligned}
\left|G_{k l}(f)\right| & =\left[G_{\mathrm{Re}}(f)^{2}+G_{I m}(f)^{2}\right]^{1 / 2} \\
\phi(f) & =a \tan \left[G_{I m}(f) / G_{\mathrm{Re}}(f)\right] .
\end{aligned}
$$

The term $\phi(f)$ of (4) corresponds to the phase difference of signal $r_{k}(t)$ and $r_{l}(t)$ for each frequency component. For the case where the source is sufficiently distant to be approximated by a plane wave at the receiver locations, the angle of incidence $\theta(f)$ for the received signal can be interpreted as $\phi(f)$ given by

$$
\phi(f)=[2 \pi f d \cos \theta(f)] / c
$$

or

$$
\theta(f)=\operatorname{acos}[c \phi(f) /(2 \pi f d)]
$$

where $c$ is the electromagnetic propagation speed in free space $\left(\sim 3 \times 10^{8} \mathrm{~m} / \mathrm{s}\right)$. The mean value of incident angles can be solved by averaging the $\theta(f)$ of all frequency components.

In practice, the continuous signal will be digitized into digital data. For digital data processing with a recording time $T$ and a sampling interval $\Delta t$ there will be $T / \Delta t=N$ data points. Thus, the discrete frequencies will be separated by $\Delta f=1 / T=$ $1 /(\Delta t . N)$, and the upper frequency limit of the analysis is $f_{c}=$ $1 /(2 \Delta t)$. Hence, for (1)-(6) the frequency increment will be $\Delta f$ with the highest frequency $f_{c}$.

\section{B. Phase Difference Analysis}

There is a dependence of the calculated $\phi(f)$ on $\theta(f)$ because of the large bandwidth and antenna separation geometry. From (5), to illustrate the relation between $\theta(f)$ and $\phi(f)$, a computer simulation has been made. The two antennas are assumed to have $d=10 \mathrm{~m}$ with the corresponding two antennas being identical. Fig. 2 shows $\phi(f)$ as a function of $f$ for several different $\theta(f)$ values and $f$ is limited to a range of $0-250 \mathrm{MHz}$. Because (5) and also (4) are trigonometric functions, the output of $\phi(f)$ varies from $\pi$ to $-\pi$ as the $\theta(f)$ varies from 0 to $\pi$. It is shown in the first and third panels that $\phi(f)$ goes through more than one cycle with increasing $f$, except for $\theta(f)=1.5108-1.6308$ radian (see the second panel). This means there are at least two frequencies that have the same $\phi(f)$ and, therefore, they provide ambiguous indication of $\theta(f)(6)$. These cycles in $\phi(f)$ are often referred to as fringes [4], [5], [7]. As the radiation source moves overhead $(\theta(f) \approx \pi / 2)$, the number of fringes decreases. However, $\phi(f)$ has a unique value or provides no ambiguity for each

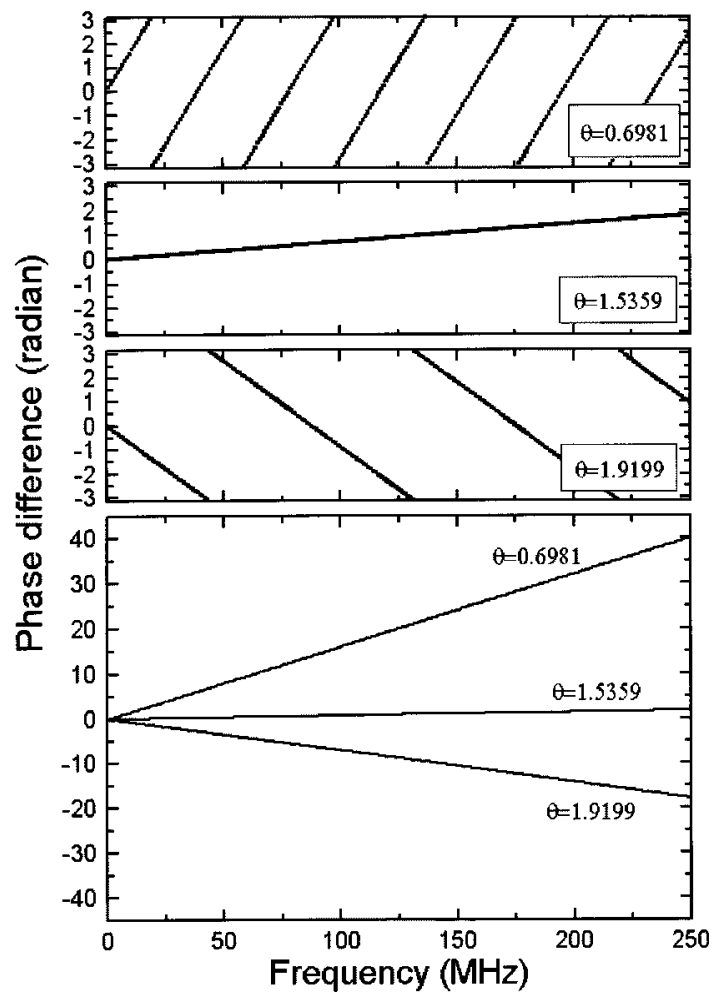

Fig. 2. The first three panels show the phase difference as a function of frequency for several incident angles. The fourth panel shows the phase difference after displacing the fringes. The unit of the incident angle is in radians.

$\theta(f)$ at any $f \leq 15 \mathrm{MHz}$, as seen from (5) for any $d \leq c / 2 f_{c}$ [4].

Note again that if the output of $\phi(f)(5)$ is not limited from $\pi$ to $-\pi, \phi(f)$ for $f \leq 250 \mathrm{MHz}$ should cover a range of about

$$
-17 \pi<\phi(f)<17 \pi
$$

or

$$
-\pi-2 m \pi \leq \phi(f) \leq \pi+2 m \pi, \quad m=8 .
$$

Equation (7) shows that $\phi(f)$ will have a maximum of eight fringes (vary from $\pi$ to $-\pi$, or $-\pi$ to $\pi$ ) as $\theta(f)$ varies from 0 to $\pi$. Therefore, it is obvious that to eliminate the ambiguity, $\phi(f)$ should have a unique value for each $\theta(f)$ for the entire frequency range. Hence, the $\phi(f)$ function should be linear as $f$ increases (see the fourth panel in Fig. 2). In our system, the procedure to remove this ambiguity was proposed as follows: 1) displacing $\phi(f)$ for the high frequencies (in our case $25-250$ MHz) over $\pm 2 n \pi$, where $n=1,2,8, m$ and 2) selecting only the $\phi(f)$ function that crosses the origin, because it is known that for $f=0, \phi(f)=0$. This process can be seen later in Figs. 7 and 8.

\section{Base Line Geometry}

Using two-antenna sensors, we only can image one-dimensional localization. To provide a two-dimensional system, the third antenna is added to determine azimuth and elevation. The first and second antennas form the first base line, and the second and third antennas form the second base line. These two base lines are perpendicular to each other. Fig. 3 shows the basic ge- 


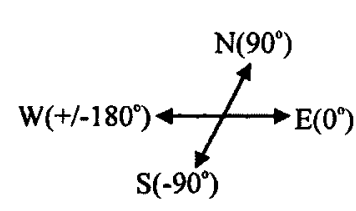

\section{Distant source}

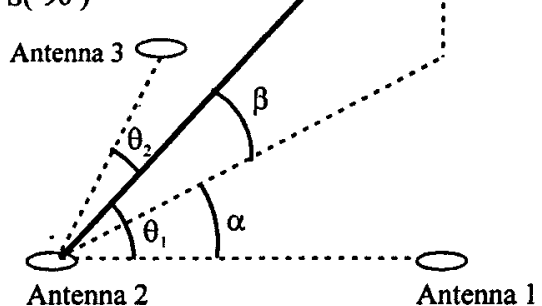

Fig. 3. Antennas 1 and 2 form the first base line and measure the incident angle $\theta_{1}$. Antennas 2 and 3 , as the second base line, measure the incident angle $\theta_{2}$. These two base lines are perpendicular to each other. The azimuth and elevation of the source can be determined from those incident angles.

ometry of a perpendicular base line interferometer. Consider a source in the northeast quadrant. Each base line provides a direction cosine between the source and the respective base line. We will use the degree unit rather than the radian unit for the explanation of angular position. Let $\theta_{1}$ and $\theta_{2}$ be the incident angles of the source for the first base line and the second base line, respectively [5]. The azimuth $(\alpha)$ and elevation $(\beta)$ of the source can be determined using the relations

$$
\cos \left(\theta_{1}\right)=\cos (\alpha) \cos (\beta)
$$

and

$$
\cos \left(\theta_{2}\right)=\sin (\alpha) \cos (\beta)
$$

By dividing (9) with (8), with the constraint $\theta_{1} \neq 90^{\circ}$ and $\beta \neq$ $90^{\circ}$, we obtain

$$
\alpha=a \tan \left[\cos \left(\theta_{2}\right) / \cos \left(\theta_{1}\right)\right]
$$

Finally, we determine $\beta$, as

$$
\beta=a \cos \left[\cos \left(\theta_{1}\right) / \cos (\alpha)\right]
$$

\section{EXPERIMENTAL SETUP}

An experiment to locate fast-moving electromagnetic sources emitted from lightning discharges was conducted in Darwin, Australia in summer 1997. Fig. 4 shows the broadband interferometer measurement system. The system employed three broadband circular flat antennas having diameter of $30 \mathrm{~cm}$ each. They were separated horizontally with the base line's length $d=10 \mathrm{~m}$ and aligned at three apexes of a square (see Fig. 3). The antennas were connected to a digitizer through coaxial cables and high-pass filters $(>25 \mathrm{MHz}$ ). The broadband lightning $d E / d t$ signals were digitized at $500 \mathrm{MHz}$ sampling rate $(\Delta t=2 \mathrm{~ns})$ using a digitizer having 8-bit resolution, which was controlled by a Personal Computer (PC) through the IEEE-488 interface bus. Because of such high digitization rate, the entire radiation from a lightning flash cannot be recorded continuously. For example, in order to record a one-second-long stream, 500 MWords memory per-channel of digitizer is required, which is

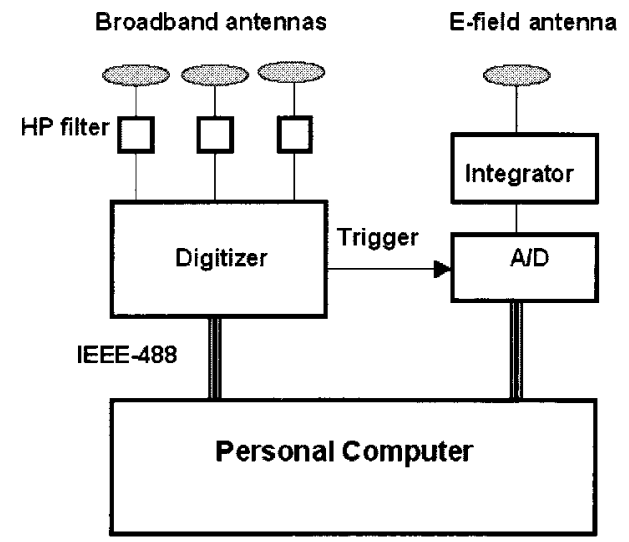

Fig. 4. Broadband measurement system.

not practical. To overcome this difficulty, we applied a sequential triggering technique for each electromagnetic pulse. The whole memory of the digitizer was divided into segments, and each segment can record one broadband electromagnetic pulse for $1 \mu \mathrm{s}$ or 502 equally spaced samples. Once the electromagnetic pulse from a lightning flash is detected and its amplitude exceeds a threshold level, the triggering circuit is turned on to record a waveform and save it as one segment. The pre-trigger setup was $50 \%$, and the minimum time between two consecutive segments was about $70 \mu$ s due to instrumentally dead time. With this technique, the system can record a maximum of 2000 segments within $1 \mathrm{~s}$. The system was operated to record radiation sources from $f=25 \mathrm{MHz}$ to $f_{c}=250 \mathrm{MHz}$. Of 502 data points sampled for one segment, only the center 256 data points $(N=256)$ are analyzed due to the fast Fourier transform (FFT) limitation. This means $T=0.5 \mu \mathrm{s}$ and $\Delta f=2 \mathrm{MHz}$.

In addition to the broadband antennas, an electric field ( $E$-field) antenna with a decay time constant of $100 \mu$ s was also equipped to measure the electric field change $(E)$. The decay time constant was set in the integrator circuit. The $E$ signal was sampled by an A/D converter having $1 \mathrm{MHz}$ sampling rate and 12-bit resolution. The A/D converter was triggered by the digitizer and connected to the same PC. This $E$ measurement was used primarily to reveal the polarity of lightning discharges and charge motions. The synchronization between the $E$ measurement and the $d E / d t$ measurement is within $1 \mathrm{~ms}$. Thus, this broadband measurement system was capable of recording $d E / d t$ and $E$ radiation from multiple impulsive events (return stroke, leader steps, cloud discharges, etc) within individual lightning flashes, with sub-microsecond time resolution, and of placing these events in the context of the flash as a whole.

The accuracy of this system has been tested on site by comparing with the narrow band system that was already established and with an all-sky video camera. The result showed that the average discrepancy for azimuth direction is less than $1^{\circ}$ with standard deviation of $4.5^{\circ}$ and for elevation direction is less than $2^{\circ}$ with standard deviation of $5.0^{\circ}[13],[14]$.

\section{RESULTS}

As a case study, we will give attention to data from a cloudto-ground lightning flash, which was detected during the cam- 

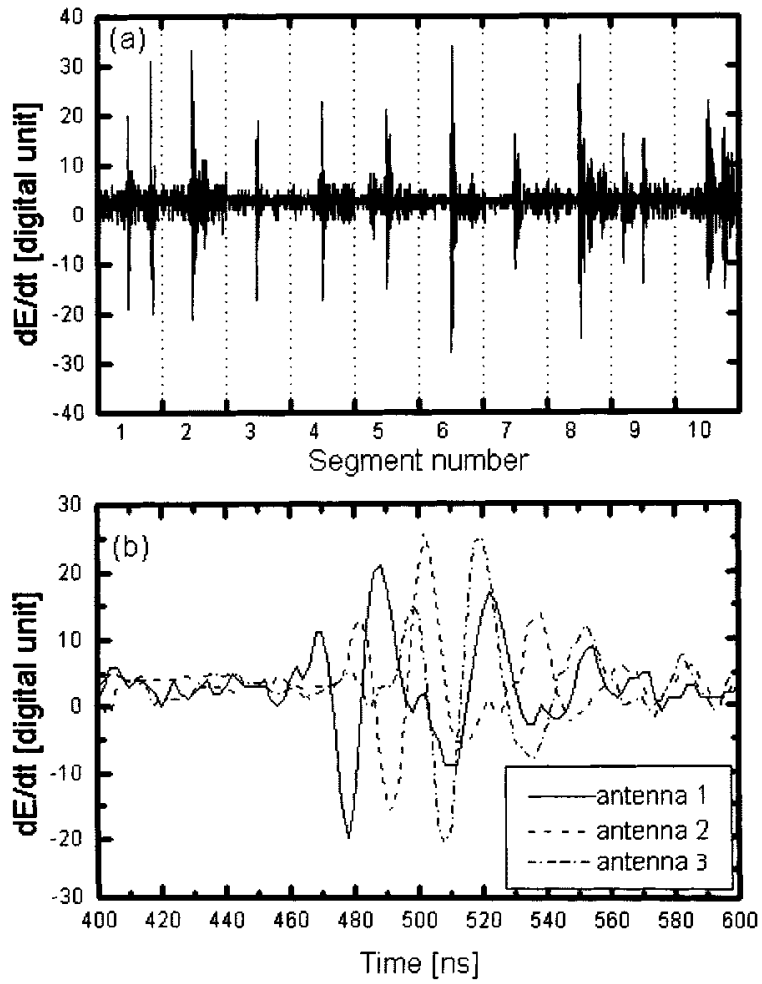

Fig. 5. Broadband signals: (a) the first ten segments of the broadband signals received by the antenna 1 and (b) expanded signal of the fifth segment.

paign on November 28, 1997 at 14:41:14 local time. This flash lasted about $400 \mathrm{~ms}$ from the beginning of broadband pulses, and 347 segments were successfully recorded. Fig. 5(a) shows the first ten segments of broadband pulses from antenna 1 and Fig. 5(b) shows the expanded scale of the broadband pulse from the fifth segment for all antennas. It is clearly seen the difference of arrival-time delay among signals. As mentioned earlier, one segment will correspond to one angular position. To simplify the next explanation of broadband interferometer processing, we will focus on the signal of this fifth segment only, and $f$ is from $25-\mathrm{MHz}$ due to the bandwidth of our system. Using the FFT, the amplitude and phase of this signal as a function of $f$ can be seen in Fig. 6 for all antennas. The $\phi(f)$ for every $f$ is shown in Fig. 7(a), and we can see fringes clearly for $f$ below $100 \mathrm{MHz}$ for each base line. For the $f$ above $100 \mathrm{MHz}$ the $\phi(f)$ seems to scatter, this might be due to the low Fourier spectrum in that frequency range [see Fig. 6(a)]. Fig. 7(b) shows the displacing process of $\phi(f)$ of the first base line (antennas 2 and 1) over $\pm 6 \pi$ only with increment $\pm 2 \pi$ (or $n=3$ ). The displacing of phase difference is continued until $n=m=8$. Then, only the $\phi(f)$ function that crosses the origin is selected, as indicated by an arrow in the Fig. 7(b). The same procedure is also applied to the second base line, and the result for the entire frequency range is shown in Fig. 8(a) for both base lines. From here, we can see that the $\phi(f)$ for $f$ above $100 \mathrm{MHz}$ still has a tendency to increase linearly as $f$ increases, although the deviation is larger than that for $f$ below $100 \mathrm{MHz}$. To find $\theta(f),(6)$ is applied to those values of $\phi(f)$ in Fig. 8(a), and the result for each base line can be seen in Fig. 8(b). By averaging $\theta(f)$ for all frequencies, then, the mean values of $\theta_{1}$ and $\theta_{2}$ are 1.15 and 2.08 radians, re-
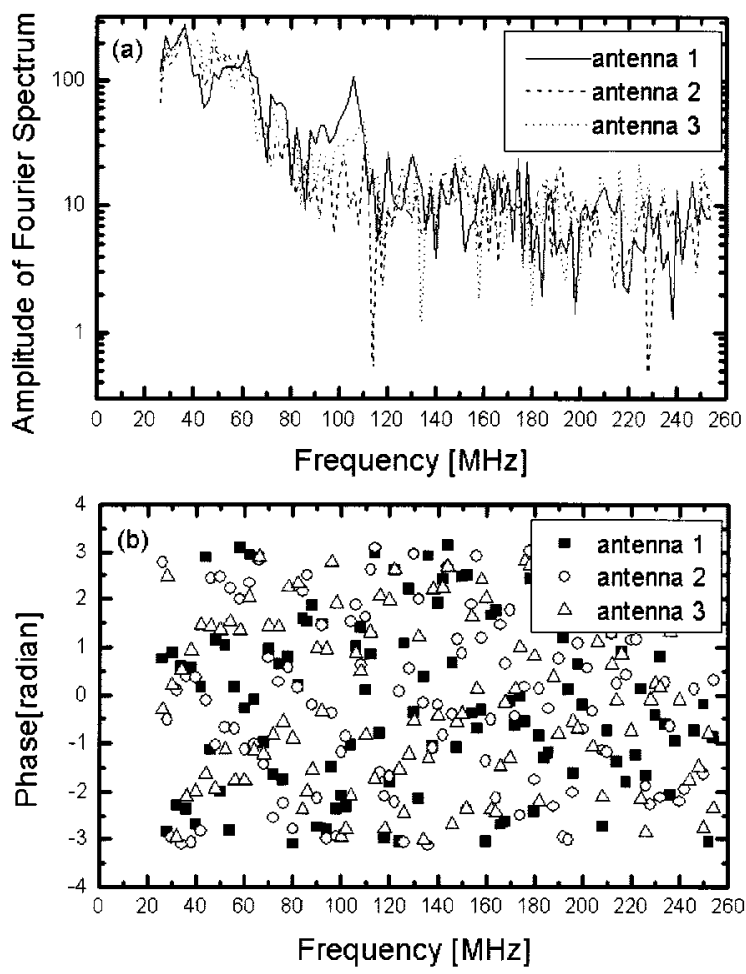

Fig. 6. Fourier spectrum of the fifth segment: (a) amplitude and (b) phase.
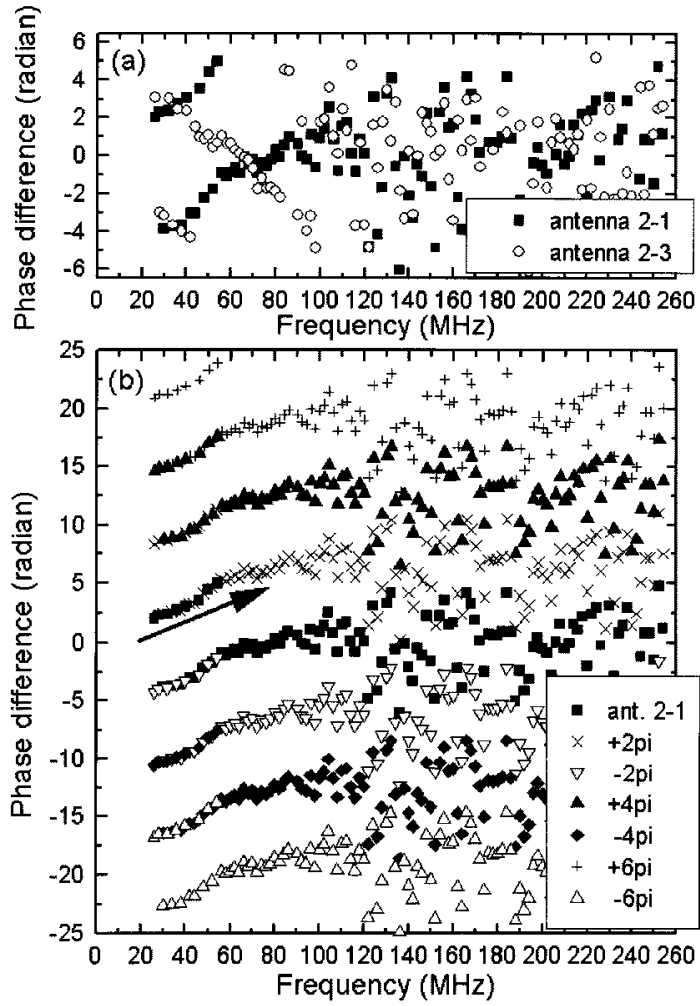

Fig. 7. Phase differences and incident angles of the fifth segment: (a) phase differences of antennas 2-1 and 2-3 show fringes for the frequency below 100 $\mathrm{MHz}$ and (b) displacing the phase difference of antennas 2-1 up to $n=3$. The arrow shows the phase difference function that crosses the origin.

spectively. By using (10) and (11), finally, the radiation source 

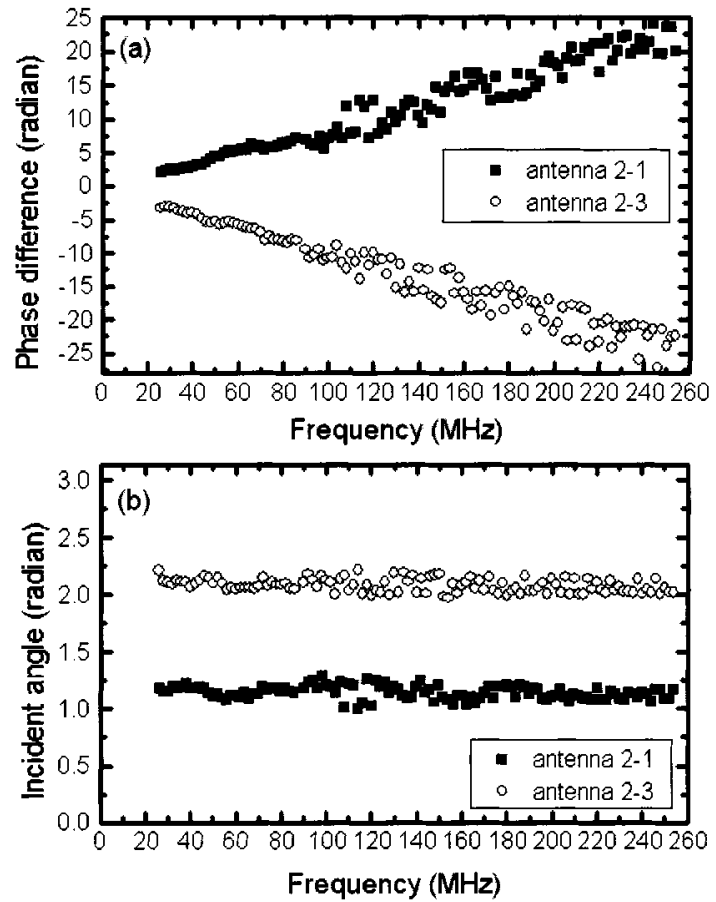

Fig. 8. (a) Phase differences after removing the fringes and (b) the incident angles for both base lines. The mean value of these incident angles is used to determine the azimuth and elevation position.

can be estimated to have $\alpha=-49.81^{\circ}$ and $\beta=50.23^{\circ}$. The position of this fifth segment can be seen later in Fig. 9 .

All successful segments can be mapped into angular-time format and azimuth-elevation format. Fig. 9 displays the activity of the flash in both formats, where a dot represents an angular position from one segment. An electric field waveform, sensed by the $E$-field antenna, and labels A, B, and $\mathrm{C}$ are also provided to simplify the explanation to this lightning event. A number of " 5 " indicates the angular position of the fifth segment that we have discussed earlier. During the A1 period, the flash began with cloud discharge activity with no significant change in electric field [Fig. 9(a)]. Strong broadband radiation was detected during the first leader progression moving downward in event A2. In this event the azimuth direction of the sources was moving from east to northeast $\left(\sim 0-60^{\circ}\right)$, as shown in Fig. 9(b), and the elevation direction moved downward starting from approximately $60^{\circ}$ [Fig. 9(c)]. In this instance the leader required about $12 \mathrm{~ms}$ to reach ground. The electric field change was negative, as seen in Fig. 9(a), indicating that negative charge was transported toward the observation site, or equivalently, that positive charge was transported away from the site. This also indicates that the lightning had negative polarity. The activity culminated with an abrupt electric field change just beyond the end of A2, caused by a return stroke. The radiation intensity dropped abruptly after the return stroke [4], [7]. That is way no pulse was detected after the return stroke for several tens of milliseconds, as seen in Fig. 9(a). During event B1, the radiation was emitted rarely and since the elevation position of this radiation was above ground level, this activity could be inside cloud discharges. The second leader progression to ground appeared during event B2 along with strong broadband radiation. Since the leader channel had approximately the same angular position
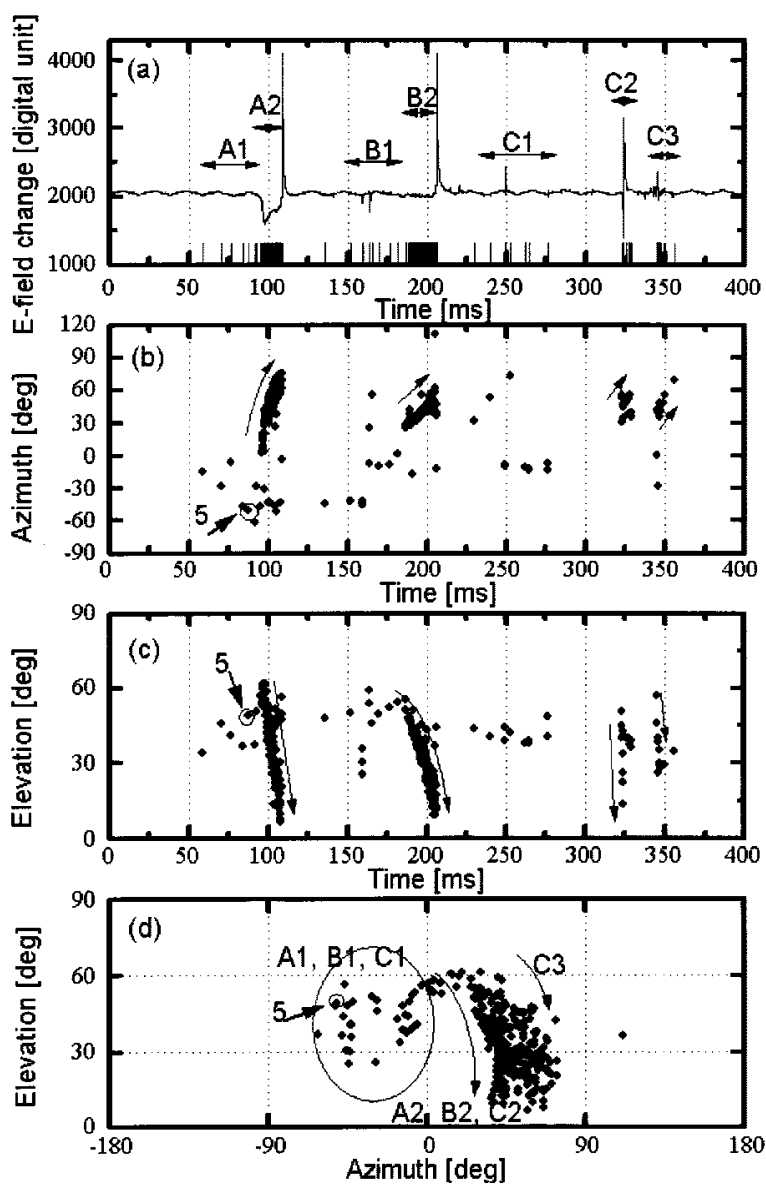

Fig. 9. Activity of a cloud-to-ground flash: (a) electric field waveform recorded by the $E$-field antenna. The series of time tags that is indicated by the thin vertical lines at the time axis shows the occurrence of the broadband signals, (b) azimuth direction of radiation sources in time sequences, (c) elevation direction in time sequences. Three lightning leaders propagating downward to ground can be identified, and (d) azimuth-elevation format. The number of " 5 " indicates the position of the fifth segment as shown in panels (b)-(d).

with the A2 event, as seen in Fig. 9(b)-(d), so the second leader channel followed the path made by the first leader. The radiation ceased at the beginning of the return stroke, whose electric field changed abruptly. This leader completed its extension within $15-18 \mathrm{~ms}$. The last activity of this flash was similar to event B1. The system recorded some radiation during event $\mathrm{C} 1$ where the position was inside the cloud. The fast field change during the very short period of within $2-4 \mathrm{~ms}$ in event $\mathrm{C} 2$ shows the third leader progression followed by a return stroke. We approximate that the path of this leader channel was also the same with the previous leaders in event A2 and B2. Such discharges propagating downward inside the clouds labeled C3 occurred after several milliseconds after the last return stroke.

\section{CONCLUSIONS}

A broadband interferometer technique for locating fast-moving electromagnetic sources emitted from lightning has been introduced and demonstrated. The basic principle of this technique is to extract the phase difference $\phi(f)$ at various frequency components of the Fourier spectra between a pair of broadband antenna sensors, and thereafter the incident 
angle $\theta(f)$ of the radiation sources can be determined. There is a dependence of the calculated $\phi(f)$ on $\theta(f)$ because of the large bandwidth and base lines geometry, and this possibly produces the ambiguous indication of source direction. Arranging $\phi(f)$ to have a unique value for the entire frequency range can eliminate this ambiguity.

The system applied a high digitization rate to overcome the large lightning frequency bandwidth, and a sequential triggering technique was used to overcome the limitation of digitizer memory. The memory of the digitizer was divided into segments. One segment can record one broadband electromagnetic pulse for $1 \mu \mathrm{s}$, and it corresponds to one angular position of the lightning radiation source. A total of 2000 segments within $1 \mathrm{~s}$ can be acquired by the system, which is enough to measure a whole lightning flash progression.

We analyzed a cloud-to-ground flash as a case study. From this flash, a total number of 347 segments had been successfully recorded within $400 \mathrm{~ms}$. The lightning discharge had negative polarity as measured by the electric field sensor. Strong broadband radiations were detected along with all leaders progression. Then, the radiation ceased and an abrupt electric field change occurred just after each leader reached the ground. All leader channels were estimated to have the same angular position, indicating that subsequent leaders followed the channel path made by the first leader to ground.

\section{ACKNOWLEDGMENT}

The authors would like to thank T. Ushio, Y. Ota, and M. Murakami for their stimulating discussions and assistance during setup of the broadband interferometer system, during the experiment, and in editing this paper.

\section{REFERENCES}

[1] D. E. Proctor, "A hyperbolic system for obtaining VHF radio pictures of lightning," J. Geophys. Res., vol. 76, pp. 1478-1489, 1971.

[2] —, "VHF radio pictures of cloud flashes," J. Geophys. Res., vol. 86, pp. 4041-4074, 1981.

[3] D. E. Proctor, R. Uytenbogaardt, and B. M. Meredith, "VHF radio pictures of lightning flashes to ground," J. Geophys. Res., vol. 96, pp. 5099-5112, 1991.

[4] C. T. Rhodes, X. M. Shao, P. R. Krehbiel, R. J. Thomas, and C. O. Hayenga, "Observation of lightning phenomena using VHF radio interferometry," J. Geophys. Res., vol. 99, pp. 13 059-13 082, 1994.
[5] C. O. Hayenga and J. W. Warwick, "Two-dimensional interferometric positions of VHF lightning sources," J. Geophys. Res., vol. 86, pp. 7451-7462, 1981.

[6] P. Richard, A. Dellanoy, G. Labaune, and P. Laroche, "Results of spatial and temporal characterization of the VHF-UHF radiation of lightning," J. Geophys. Res., vol. 91, pp. 1448-1260, 1986.

[7] X. M. Shao, P. R. Krehbiel, R. J. Thomas, and W. Rison, "Radio interferometric observations of cloud-to-ground phenomena in Florida," $J$. Geophys. Res., vol. 100, pp. 2749-2783, 1995.

[8] M. Le Boulch, J. Hamelin, and C. Weidman, "UHF-VHF radiation from lightning," in Lightning Electromagnetic, R. L. Gardner, Ed. New York: Hemisphere, 1990, pp. 211-256.

[9] X. M. Shao, D. N. Holden, and P. R. Krehbiel, "Broadband radio interferometry for lightning observation," Geophys. Res. Lett., vol. 23, pp. 1917-1920, 1996.

[10] T. Ushio, Z.-I. Kawasaki, Y. Ohta, and K. Matsuura, "Broad band interferometric measurement of rocket triggered lightning in Japan," Geophys. Res. Lett., vol. 24, pp. 2769-2772, 1997.

[11] R. Mardiana, Y. *Ohta, M. Murakami, T. Ushio, Z. Kawasaki, and K. Matsuura, "A broadband radio interferometer for observing lightning discharge processes," J. Atmos. Electricity, vol. 18, pp. 111-117, 1998.

[12] A. G. Piersol, "Time delay estimation using phase data," IEEE Trans Acoust., Speech, Signal Processing, vol. ASSP-29, pp. 471-477, June 1981.

[13] M. Murakami, Z. Kawasaki, Y. Ota, R. Mardiana, H. Isoda, and T. Ushio, "Comparison between broad band and narrow band interferometer for lightning observation," Inst. Elect. Eng. Jpn., vol. 119, pp. 807-812, 1999.

[14] Z. Kawasaki, R. Mardiana, and T. Ushio, "Broadband and nNarrowband interferometers,", submitted for publication.

Redy Mardiana was born in Indonesia in 1968. He received the B.S. and M.S. degrees in electrical engineering from Bandung Institute of Technology, Indonesia, in 1992 and 1997, respectively. he is currently pursuing the Ph.D. degree in the Department of Electrical Engineering, Osaka University, Osaka, Japan.

From 1993 to 1994, he was with Forshungzentrum, Juelich, Germany. His main interest is in lightning detection.

Zen Kawasaki (S'75-M'78) was born in Japan in 1949. He received the B.S., M.S., and Dr.Eng. degrees in communication engineering from Osaka University, Osaka, Japan, in 1973, 1975, and 1978, respectively.

In 1989, he joined the Department of Electrical Engineering, Osaka University, where he is currently an Associate Professor. His research interests mainly concern the observations of lightning discharges and the diagnosis techniques of power apparatus.

Dr. Kawasaki is a Member of American Geophysical Union, the Institute of Electrical and Electronics Engineers, the Institute of Electrical Engineers of Japan, and the Atmospheric Society of Japan. 